\title{
Small Molecule Tyrosine Kinase Inhibitors for the Treatment of Intestinal Inflammation
}

\author{
Maninder Sidhu, PhD ${ }^{1}$, Carmen Alonso Cotoner, MD, $\mathrm{PhD}^{1}$, Bayasi Guleng, $\mathrm{PhD}^{1}$, Seiji \\ Arihiro, MD, $\mathrm{PhD}^{1}$, Sunyoung Chang, $\mathrm{PhD}^{1}$, Kenneth W. Duncan, $\mathrm{PhD}^{2}$, Alfred M. Ajami, \\ $\mathbf{P h D}^{2}$, MyDoanh Chau, PhD $^{2}$, and Hans-Christian Reinecker, $\mathbf{M D}^{1}$ \\ ${ }^{1}$ Department of Medicine, Gastroenterology Unit, Massachusetts General Hospital and Harvard \\ Medical School, Boston, Massachusetts \\ ${ }^{2}$ Antisoma, Inc., Cambridge, Massachusetts
}

\section{Abstract}

Background-We developed a series of dendritic cell autoimmune modulators (DCAMs) based on small molecule Flt3 receptor tyrosine kinase inhibitors (TKIs) for the inhibition of intestinal inflammation and oral delivery.

Methods-DCAMs were administered orally during and after induction of dextran sodium sulfate (DSS)-induced colitis. Dendritic cell recruitment and inflammatory responses were determined in the mucosal immune system during acute intestinal inflammatory responses and mucosal recovery. Bone marrow-derived macrophages were utilized to define the mechanisms by which DCAMs can modify responses to microbial signals.

Results-Oral doses of DCAMs prevented severe weight loss and mucosal inflammation associated with DSS colitis in mice. The presence of DCAMs increased the number of $\mathrm{CD} 11 \mathrm{c}^{+} \mathrm{PDCA} 1^{+}$dendritic cells, induced interleukin (IL)-10 expression, and reduced inflammatory cytokine expression in the mucosal immune system. Surprisingly, DCAMs regulated innate immune responses in macrophages resulting in the inhibition of tumor necrosis factor alpha (TNF-a) production and the induction of IL-10 expression during Toll-like receptormediated signaling.

Conclusions-We identified two new imidazoacridinone derivatives that protect mice from severe colitis and promote mucosal recovery by enhancing protective cytokine production while inhibiting proinflammatory stimuli during microbial recognition. These compounds are promising candidates for further development into potent orally available drugs for the prevention of colitis and promotion of mucosal recovery.

\section{Keywords}

Flt3 receptor tyrosine kinase inhibitors; inflammatory bowel diseases; Crohn's disease; ulcerative colitis; novel treatment; mucosal recovery; dendritic cells; TNF-a; IL-10

\section{Introduction}

The defense response to microbiota in the lumen of the intestine is required to maintain health and to overcome disease. Crohn's disease (CD) and ulcerative colitis (UC) are

Correspondence to: Dr. Hans-Christian Reinecker, MD, Center for the Study of Inflammatory Bowel Diseases, Massachusetts General Hospital and Harvard Medical School, Gastrointestinal Unit, Jackson Bldg. R711, Fruit St., Boston MA 02114, hans-

christian_reinecker@hms.harvard.edu. 
noninfectious, chronic, and relapsing inflammatory diseases of the gastrointestinal (GI) tract characterized by injury to the barrier function of the intestine. This is the result of an exaggerated defense response to the intestinal microbiota leading to recurrent and longlasting episodes of diarrhea and abdominal pain. ${ }^{1-4}$ None of the current available treatments specifically target the reason for the disruption of inflammatory or inhibitory regulation underlying the diverse and multifactorial diseases that we understand as inflammatory bowel diseases (IBD). Initial recognition of intestinal microbiota is mediated by pattern recognition receptors (PRRs) such as nucleotide binding oligomerization domain (NOD)-like receptors (NLRs). ${ }^{5,6}$ Toll-like receptors (TLRs), ${ }^{7,8}$ Rik, and MDA5 receptors ${ }^{9}$ recognize conserved microbial structures known as pathogen-associated molecular patterns (PAMPs). The combination of these signaling pathways controls innate immune responses to microbial effectors, which include the activation of nuclear factor kappa B (NF-jB)-dependent proinflammatory mediators such as tumor necrosis factor alpha (TNF-a), but also initiate signaling circuitry stimulating interleukin (IL)-10 expression to control inflammation. This coordination of different inhibitory and inflammatory regulatory pathways is of importance for the control of innate immune responses at mucosal interphases, where a multitude of microbial challenges require a controlled host defense response. Thus, treatment approaches for the control of IBD have to balance host defense responses without interrupting immune signaling responsible for the secretion of inhibitory factors required for mucosal healing.

Receptor tyrosine kinases (RTKs) constitute a family of proteins involved in growth and developmental processes. Fms-like tyrosine kinase ligand (Flt3) is an RTK that was originally reported in 1991 as a gene with similarities to fms, c-kit, and pdgfr. ${ }^{10} \mathrm{Flt3L}$ promotes the survival, commitment, and differentiation of hematopoietic progenitors to the dendritic cell (DC) lineage ${ }^{11}$ but also regulates innate immune cell function in the periphery. ${ }^{12,13}$ Flt $3 \mathrm{~L}$ treatment can indirectly expand DC-induced peripheral naturally occurring regulatory $\mathrm{T}$ cells (Tregs) ${ }^{14}$ and loss of regulatory $\mathrm{T}$ cells increases DC division by a Flt3-dependent mechanism. ${ }^{15}$ Furthermore, Flt3L inhibits IL-10 expression by Foxp3 ${ }^{+}$ Tregs $^{16}$ and therefore may be directly involved in the control of innate immune responses. We developed a new series of Flt3 receptor tyrosine kinase inhibitors (TKIs) based on the parent compound, Symadex (formerly C1311). Symadex demonstrated activity in models of colorectal, colon, and breast cancer ${ }^{17}$ and was demonstrated to be active in the Experimental Autoimmune Encephalomyelitis (EAE) model by promoting permissive remyelination of spinal cord. ${ }^{18}$ Further modifications of the imidazoacridinone scaffold and its functional decorations, as shown in Figure 1, resulted in two noncytotoxic compounds with oral bioavailability which we found had a remarkable ability to inhibit mucosal inflammation and to promote mucosal recovery. We demonstrate that the two identified dendritic cell autoimmune modulators (DCAMs) can enhance IL-10 secretion by macrophages in the context of microbial recognition while inhibiting proinflammatory cytokine secretion.

\section{Materials and Methods}

\section{Induced Mouse Model of Dextran Sulfate Sodium (DSS) Colitis}

Wildtype (C57BL/6) mice were purchased from the Jackson Laboratory (Bar Harbor, ME). This study was carried out in strict accordance with the recommendations in the Guide for the Care and Use of Laboratory Animals of the National Institutes of Health. The protocol was approved by the Committee on the Ethics of Animal Experiments of the Massachusetts General Hospital (Permit Number: A3596-01). Female mice (n 1/4 5/group) of 4-6 weeks of age and a weight range of 17-19 g were used for the study. Mice were given 4\% DSS (MP Biomedicals, Solon, $\mathrm{OH}$ ) in drinking water for 5 days and normal drinking water was continued thereafter. Mice were weighed daily for 12 days, weight was recorded, and percent weight loss was calculated at the end of the study. Tissues including colon, mesenteric lymph nodes (MLNs), and spleen were harvested for further analysis. 


\section{Administration of Compounds}

Powdered compounds (DCAM-3 and DCAM-4) were dissolved in the formulation mixture (PEG400: Tween20: Transcutol, Sigma-Aldrich, St. Louis, MO) to obtain a stock concentration of $20 \mathrm{mg} / \mathrm{mL}$. The compounds were diluted in vegetable oil for a dose of 50 $\mathrm{mg} / \mathrm{kg}$. In independent experiments, two oral doses of compounds or oil control were given on Day 3 and Day 5 or three doses of the compound or oil control were given orally on Day 1, Day 3, and Day 5, respectively.

\section{Gross Examination and Histological Analysis}

Mice in all of the treatment and control groups were sacrificed at the end of the study and the colons, MLNs, and spleens were harvested. The harvested tissues were examined and scored for enlarged lymph nodes or spleen and thickening of the colon. The MLNs were stored at $-80^{\circ} \mathrm{C}$ in $1 \mathrm{~mL}$ of Trizol for RNA isolation and the colons were further processed for histology. Briefly, the colons were inverted and cleaned in phosphate-buffered saline (PBS). The cleaned colons were inverted back and cut into three sections (proximal, middle, and distal) and were fixed in 10\% formalin. Tissue cross-sections were made and slides were stained using hematoxylin and eosin (H\&E). The slides were scored for severity of inflammation or absence thereof. Photographs of the representative sections were taken using Nikon eclipse 50i microscope (Melville, NY).

\section{Histological Scoring}

Histological scoring was performed on operator-blinded sections using a previously validated scoring system. ${ }^{19}$ The colon was divided into proximal, middle, and distal portions and were fixed in 10\% formalin. Sections 5-8 $\mathrm{lm}$ thick were prepared and subjected to staining with $\mathrm{H} \& \mathrm{E}$. Three independent parameters were measured (severity of inflammation, depth of injury, and crypt damage) and were scored from 0 (normal) to 3 (severe, transmural injury/inflammation with entire crypt and epithelium loss). The changes were also quantified as a percentage of involvement by the disease process, i.e., the score of the parameter was multiplied by a factor reflecting the percentage of tissue involvement. Each section was then scored for each feature separately by establishing the product of the grade and the percentage involvement and added to a sum with a maximum possible score of 40 . The score was plotted for each group of treated mice and the error bars represent standard deviation of the mean values.

\section{Cytokine Measurement}

Cell culture supernatant samples were analyzed for cytokine production using the Procarta Mouse Cytokine Profiling Kit (Panomics, Affymetrix, Santa Clara, CA). Briefly, the 96well filter plate was pre-wet with reading buffer. Premixed antibody beads were added and buffer was removed by vacuum filtration. Samples were added to the wells and incubated on a shaker at $600 \mathrm{rpm}$ for 60 minutes at room temperature (RT). After washing, premixed detection antibodies were added to the plate and incubated on a shaker at $600 \mathrm{rpm}$ for 30 minutes at RT. Streptavidin-PE was added to the plate and incubated on a shaker at $600 \mathrm{rpm}$ for 30 minutes at RT. After washing, the plate was prepared for analysis on a Luminex (Austin, TX) instrument by adding reading buffer to all of the wells. Data were collected and analyzed by using the designated Luminex acquisition software and Microsoft Excel.

\section{In Vitro Macrophage Cultures}

Bone marrow derived macrophages (BMMs) were isolated and cultured as described previously.20 Briefly, the femurs and tibias of wildtype mice were harvested, muscle wasremoved, and the bones were cleaned with $70 \%$ ethanol. The bone marrow was flushed out of the bones using 10\% RPMI medium supplemented with L-glutamine heat-inactivated 
fetal calf serum and penicillin-streptomycin (CellGro, Mediatech, Herndon, VA). The BMMs were treated with ACK Lysis buffer (BioWhittaker, Lonza, Walkersville, MD) to lyse the red blood cells. Cells were washed, counted using a hemocytometer, and the concentration was adjusted to $2 \times 10^{6}$ cells $/ \mathrm{mL}$ in the $10 \%$ RPMI medium containing MCSF $(20 \mathrm{ng} / \mathrm{mL})$. The medium was changed every 2 days and replaced with fresh M-CSF supplemented RPMI medium. Fully differentiated macrophages were collected on Day 6 by treating the cells with $10 \mathrm{mM}$ EDTA-PBS solution. The cells were washed, resuspended in fresh RPMI medium, counted, and used for the in vitro studies. The compounds were dissolved in DMSO at a concentration of $5 \mathrm{mM}$. BMM $\left(1 \times 10^{5}\right.$ cells $\left./ \mathrm{mL}\right)$ were stimulated with lipopolysaccharide (LPS; $1 \mathrm{lg} / \mathrm{mL}$ ), CpGODN1826 (5 lM), and exposed to respective compounds at a final concentration of $100 \mathrm{nM}$ for 24 hours at $37^{\circ} \mathrm{C}$. Culture supernatants were collected for analysis of cytokine production. Macrophages were suspended in RLT (RNA lysis buffer, Qiagen, Valencia, CA) for RNA isolation and samples were prepared for quantitative polymerase chain reaction (PCR) for analysis of cytokine gene expression.

\section{RNA Isolation and Quantitative PCR}

The MLNs were harvested at the end of experiment and suspended in Trizol reagent (Invitrogen, Carlsbad, CA) and stored at $-80^{\circ} \mathrm{C}$ for RNA isolation. On the day of isolation, MLNs were thawed and the tissue was disrupted using a homogenizer (Mini-Bead Beater, BioSpec Products, Bartsville, OK). Total RNA was extracted using Trizol reagent and the samples were treated with DNase according to the manufacturer's protocol (Promega, Madison, WI). RNA was cleaned further using the RNeasy Mini kit (Qiagen). Template cDNA was synthesized by using the iScript cDNA synthesis kit (Bio- Rad, Hercules, CA) and quantitative PCR was performed using the iQ SYBR Green Supermix kit using the BioRad CFX96 thermal cycler (Bio-Rad). The normalized gene expression $(\Delta \Delta \mathrm{Ct})$ values were calculated with GAPDH as a reference gene in all samples plotted as standard errors of mean values. The following oligonucleotides were used as primers: mIL- $1 \beta$ forward, $5^{\prime}$ GCAACTGTTCCTGAACTCAACT and reverse, $5^{\prime}$-ATCTTTTGGGGTCCGTCAACT; mGAPDH forward, 5'TGGCAAAGTGGAGATTGTTGCC and reverse, $5^{\prime}$ -

AAGATGGTGATGGGCTTCCCG; mIL-10 forward, 5' -TA

AGGGTTACCTGGGTTGCCAAG and reverse, $5^{\prime}$-CAAATGCTCCTTGATTTCTGGGC; mIL-6 forward, $5^{\prime}$-TAGTCCTTCC TACCCCAATTTCC and reverse, $5^{\prime}$ -

TTGGTCCTTAGCCACTCCTTC; mTNF-a forward, $5^{\prime}$ CCCTCACACTCAGATCATCTTCT and reverse, 50-GCTACGACGTGGGCTACAG.

\section{Isolation of Colonic DCs and Lamina Propria T Cells}

Colons harvested at the end of the study were used for colon DC isolation and small intestines were used for T-cell isolation. The colon was inverted on polyethylene tubes (Becton Dickinson, Franklin Lakes, NJ), washed three times in calcium- and magnesiumfree PBS (BioWhittaker), and mucus was removed using $1 \mathrm{mM}$ dithiothreitol (DTT; SigmaAldrich). The intestinal epithelium was eluted with $30 \mathrm{mM}$ EDTA at RT, followed by digestion of the tissue with $108 \mathrm{U} / \mathrm{mL}$ type IV collagenase (Sigma-Aldrich) in Dulbecco's odified Eagle's medium (DMEM; Mediatech, Manassas, A) containing 5\% fetal calf serum for 90 minutes at $37^{\circ} \mathrm{C}$ in $5 \% \mathrm{CO} 2$ incubator. The digested tissue was gently shaken or 10 minutes at RT and then passed through a 10- $\mu$ m nylon trainer and washed with DMEM. Finally, OptiPrep (Axis-Shield, slo, Norway) density centrifugation (density is $1.055 \mathrm{~g} / \mathrm{mL}$ ) ields colon DCs. Lamina propria T cells were isolated similarly xcept that the Optiprep density gradient (Sigma-Aldrich) centrifugation was performed at a density of $1.084 \mathrm{~g} / \mathrm{mL}$.

\section{Flow Cytometry}

Cells isolated from the colon and lamina propria were further nalyzed by flow cytometry. Cells were incubated with $10 \%$ onkey serum and Fc block (BD Pharmingen, San Jose, CA) 
for 0 minutes at $4{ }^{\circ} \mathrm{C}$ and then stained with fluorescent conjugated ntibodies. APC conjugated antimouse CD11c, PE conjugated ntimouse PDCA1, Per-CP conjugated antimouse CD11b, and ITC conjugated antimouse CD103 (BD Pharmingen) were used or FACS staining. Cells were analyzed on a FACSCalibur ytometer (BD Bioscience, San Diego, CA) and further analyzed y using FlowJo software (Tree Star, Ashland, OR).

\section{Statistical Analysis}

Statistical analysis was carried out by two-way analysis of variance (ANOVA) followed by Bonferroni post-tests. $\mathrm{P}<0.05$ was considered statistically significant.

\section{Results \\ DCAMs Are Selective, Orally Bioavailable Inhibitors of FIt3 RTK with a Favorable Drug Development Profile}

DCAMs 3 and 4 resulted from a lead qualification and target optimization campaign to recraft the molecular scaffold of Symadex, the pathfinder structure, in order to preserve the "core," shown in Figure 1, while varying the "head" and "tail" moieties with isosteric replacements or well-known drug-like fragments from the RTKi chemical repertory. The development effort was guided initially by a structural biology model in which new constructs were selected in silico as putative hits based on their similarity to the docking poses and active site affinities of the pathfinder molecule. These were then synthesized and qualified by bioassay against Flt3 wildtype and mutant (D835Y) isoforms. Analogs showing average combined $\mathrm{IC}_{50}$ values less than $25 \mathrm{nM}$ were then subjected to a screening cascade for lead optimization, in part as described by Bürli et al. ${ }^{21}$ The overarching goal in this effort was to eliminate the cytotoxic, mutagenic, hERG inhibitory, and metabolic liabilities associated with Symadex. Eight advanced leads, including DCAMs 3 and 4, emerged from within the cohort of molecules synthesized. One or more of these will be considered for clinical development upon meeting the safety and regulatory requirements for investigation in human subjects. As shown in Table 1, DCAMs 3 and 4 are potent inhibitors of Flt3 and its point mutant, with a specificity that is characterized by a narrow set of off-target hits, clustered predominantly among the Class III receptor tyrosine kinases to which Flt3 belongs. Notably, the DCAMs do not inhibit epidermal growth factor receptor (EGFR), fibroblast growth factor receptor (FGFR), vascular endothelial growth factor receptor 2 (KDR), c-kit, Met, Ret, or the insulin receptor-related kinases $\left(\mathrm{IC}_{50}>5000 \mathrm{nM}\right)$. They are nonmutagenic by Ames Test, raise no significant alerts in terms of interaction with a standard panel of cell receptors and ion channels, and pass the accepted hurdles for "drugability," metabolic stability in human hepatocytes, bioavailability, pharmacokinetics, and metabolic disposal in vivo (mouse). In addition, their pharmacokinetics were found to be dose-proportional, in contradistinction to those of Symadex. Metabolism did not appear to involve CYPs in the liver and is attributable predominantly to cytosolic oxidation by aldehyde oxidase (Antisoma, unpubl. obs.).

\section{DCAMs Prevent Intestinal Inflammation and Promote Mucosal Recovery in the Mouse Model of DSS-induced Colitis}

To determine the ability of DCAM3 and DCAM4 to inhibit intestinal inflammation maintained by innate immune responses to epithelial barrier disruption we utilized the DSSmediated colitis model. For the study, female C57BL/6 mice ( $n=5 /$ group) at $4-6$ weeks of age and weight range from 17-19 g were selected and exposed to 4\% DSS colitis in the drinking water for 5 days to induce severe colitis and allowed to recover for 6 days to study mucosal recovery. In the first set of experiments, mice were given three oral doses of compounds on Day 1, Day 3, and Day 5 and weight was monitored throughout the course of the study. We found that oral gavages with either DCAM3 or DCAM4 were efficient in 
inhibiting weight loss during DSS-induced colitis when given concurrently with the DSS (Fig. 2A). In the vehicle control group, mice lost $10 \pm 2 \%$ of weight by Day 6 compared to 6 $\pm 1 \%$ weight loss in the DCAM3 and DCAM4 treated mice, respectively. After removal of DSS from the drinking water, control mice continued to lose weight with $15 \pm 1 \%$ weight loss at Day 8 while DCAM-treated mice lost $9 \pm 1 \%$ in the same time period. Although by Day 11 the vehicle-treated mice had gained some weight, the percent weight loss $(9 \pm 1 \%)$ was still higher compared to the DCAM-treated mice $(2 \pm 1 \%)$. DCAM treatment enhanced the process of mucosal healing and recovery from intestinal inflammation accompanied by steady weight recovery.

Furthermore, DCAMs were also able to significantly inhibit intestinal inflammation and promote intestinal recovery after the onset of colitis (Fig. 2B). Mice were treated with compounds DCAM3 and DCAM4 3 days after the beginning of the DSS treatment and on Day 6 after discontinuation of DSS. By Day 6 the percent weight loss in the vehicle control group was $8 \pm 1 \%$ compared to $3 \pm 1 \%$ in DCAM3 and $5 \pm 1 \%$ in DCAM4-treated mice. In the treatment groups, weight loss was significantly less by Days 9 to 11 , while mice that received vehicle control showed a high percentage of weight loss $(3 \pm 1 \%)$ with signs of severe mucosal inflammation persisting for several days as a sign of exacerbating colitis.

In the macroscopic analysis, we found that mice treated with the compounds DCAM3 or DCAM4 presented with few signs of mucosal inflammation compared to the mice treated with vehicle alone, which presented with thickened colons, enlarged spleens, and MLNs (Fig. 3A,B) by Day 11. The histological examination was performed in a blinded fashion using a previously validated scoring system. 19 The scores in the vehicle-treated group were significantly higher for the severity of inflammation, depth of injury, and crypt damage, with scores being 9, 8, and 9.6, respectively. Comparatively, DCAM4-treated mice had scores of 4, 4.6, 3.3, and DCAM3-treated mice had scores of 4.3, 4.3, and 4.6, respectively, indicating mild inflammation in the colons of DCAM treated mice (Fig. 4A). Mice treated with vehicle alone presented with transmural leukocyte infiltration with tissue destruction indicating severe persistent colitis. In contrast, low or no cellular infiltration but enhanced goblet cell numbers and intact intestinal epithelium were observed in the colon sections of mice treated with DCAM3 or DCAM4 (Fig.4B).

\section{DCAMs Regulate Macrophage and DC Subset Composition in the Mucosal Immune System}

Both compounds demonstrated distinct roles in modulating macrophage and DC subspecifications in the lamina propria of the colon. We found a significant increase in the percentage of total CD11 ${ }^{+} \mathrm{PDCA}^{+}$cells in the colon of DCAM3-treated mice but not in the DCAM4 treated mice (Fig. 5A,C). The percentage of CD11 ${ }^{+}$PDCA $1^{+}$cells in the DCAM3-treated mice was $10.6 \pm 1 \%$ compared to DCAM4-treated $(6 \pm 0.5 \%)$ and vehicle control treated mice $(5 \pm 2 \%)$. In contrast, our analysis revealed that the DCAM4-treated mice had a decreased percentage of $\mathrm{CD} 11 \mathrm{~b}^{+} \mathrm{F} 4 / 80^{+}$macrophages $(9 \pm 0.5 \%)$ in the colon compared to DCAM3 $(13.7 \pm 2 \%)$ or vehicle-treated mice (13 $\pm 2 \%)$ (Fig. 5B,C).

\section{The Compounds Regulate In Vivo Cytokine Gene Expression}

To dissect the mechanism by which DCAMs modulate intestinal inflammation and recovery, we determined cytokine expression in the mucosal immune system during treatment with DCAM3 or DCAM4. MLNs were harvested from mice treated with the compounds or vehicle control to analyze the expression of IL-10, IL- $1 \beta$, IL- 6 , and TNF- $\alpha$ as key regulators of mucosal inflammation. Mice treated with DCAM3 showed a significant reduction and DCAM4-treated mice showed a 6-fold reduction in the expression of TNF-a compared to the control vehicle-treated group (Fig. 6). In contrast, the expression of IL-10 was significantly higher in the MLNs from mice treated with the compounds. A 2-3-fold 
increase in IL-10 gene expression was observed after treatment with both DCAMs compared to the vehicle controls. Although the gene expression levels of IL- $1 \beta$ or IL- 6 appeared reduced in DCAM-treated mice, the comparison did not demonstrate statistical significance.

\section{DCAMs Control Toll-like Receptor Mediated Innate Immune Responses}

Macrophages, monocytes, and DCs are a major source of proinflammatory cytokines that activate both local and systemic immune responses in response to TLR signaling. We determined the ability of the compounds to regulate TLR4 and TLR9 signal transduction in BMMs. As shown in Figure 6, proinflammatory cytokine production of IL-6 and TNF-a by $\mathrm{CpG}$ activated BMMs was significantly reduced during exposure to DCAM3 or DCAM4 (Fig. 7A). Production of IL-6 by BMMs in response to TLR9 activation was 40-fold and 80fold less in the presence of DCAM3 and DCAM4, respectively, compared to IL-6 levels in the absence of the compounds. TNF-a production by BMMs in response to TLR9 activation was 7-fold and 18-fold less in presence of DCAM3 and DCAM4, respectively, in comparison to levels in the absence of the compounds. Furthermore, IL- $1 \beta$ gene expression was significantly reduced by 2 -fold in the LPS-activated BMMs exposed to either compound (Fig. 7B). In contrast, both compounds significantly enhance IL-10 production by activated peritoneal macrophages alone by 50 -fold (Fig. 7C). In addition, during TLR4 signaling, DCAM3 and DCAM4 significantly enhanced IL 10 gene expression by 2 -fold compared to the BM stimulated by LPS alone. However, DCAMs were unable to further enhance IL-10 expression in CpG-activated BMMs. Thus, our results indicate that DCAMs can directly modulate innate immune responses of macrophages by inhibiting proinflammatory cytokine production in response to TLR4 and TLR9 signaling while enhancing LPS induced IL-10 expression as a key mediator required for mucosal homeostasis.

\section{Discussion}

Our study demonstrates the ability of two novel small molecule Flt3 receptor TKIs to control mucosal inflammation and to promote mucosal recovery in the DSS-induced mouse model of colitis when given as oral doses. The inhibition of mucosal inflammation may be due to the ability of DCAMs to regulate host defense responses of macrophages and DCs during microbial recognition. Microbial pattern recognition by TLR ligands include not only proinflammatory gene expression but also the induction of IL-10 gene expression to control the immune response. ${ }^{22}$ DCAMs represent a new class of small molecule inhibitors that have the unique ability to inhibit proinflammatory innate immune responses while enhancing the inhibitory arm of mucosal immune responses. These compounds may thus have significant therapeutic potential for combating microbial-induced and maintained chronic mucosal inflammation in CD and UC.

The two compounds differently regulated innate immune cell subsets in the mucosal immune system during inflammation. Increased numbers of $\mathrm{CD} 11 \mathrm{c}^{+} \mathrm{PDCA} 1^{+}$cells were observed in colons of mice treated with DCAM3. Studies have shown that mucosal $\mathrm{CD} 11 \mathrm{c}^{+} \mathrm{PDCA} 1^{+} \mathrm{pDC}-$ like cells promote tolerance through IL-10 and/or TGF- $\beta$ release, thereby supporting the de novo differentiation of Tr-type regulatory $\mathrm{T}$ cells ${ }^{23} \mathrm{In}$ contrast, mice treated with DCAM4 showed a decrease in the numbers of CD11 ${ }^{+} \mathrm{F} 4 / 80^{+}$cell population in the lamina propria. Mononuclear phagocyte numbers increase in the inflamed intestinal mucosa of patients with active IBD and a significant positive correlation has been noted between the number of infiltrating intestinal mononuclear phagocytes and the degree of crypt inflammation in UC. ${ }^{24}$ Further studies will help delineate the differences in the mechanisms of action of the two compounds and aid in the elucidation of additional application for inflammatory disease with distinct underlying immunoregulatory deficiencies. 
Both DCAMs are able to inhibit proinflammatory cytokine production by macrophages linked to the pathophysiology of IBD such as TNF-a, IL-6, and IL-1 $\beta .{ }^{25}$ DCAMs inhibited TLR4 and TLR9-mediated production of IL- 6 and TNF-a by macrophages. TNF-a and IL-6 are proinflammatory cytokines that are key mediators in the inflammatory process in IBD. Clinical studies have reported a dramatic improvement in IBD patients treated with antiTNF-a therapy, ${ }^{26}$ although side effects of anti TNF-a treatment in IBD have emerged which include the reactivation of viral or bacterial infections. ${ }^{27}$ Furthermore, anti-TNF- $a$ treatment-resistant patient groups have emerged. ${ }^{28}$ Also reducing IL-6 levels with IL-6R $\mathrm{mAb}$ treatment in patients with $\mathrm{CD}$ has been shown to be effective in controlling inflammation. ${ }^{29}$ While both DCAMs inhibited TNF- $a$ and enhanced IL-10 expression in vivo and in vitro, we observed differences between the two compounds in the regulation of IL- $1 \beta$ in vivo. This may be due to distinct differences in bioavailability and disposal (clearance and volume distribution) (Table 1), since both compounds inhibited in vitro LPS or CpG-induced IL- $1 \beta$ expression in BMMs. Increased amounts of IL-1 $\beta$ mRNA have been observed in the inflamed mucosa of a majority of IBD patients and high IL-1 $\beta$ concentrations have been detected in the mucosa of animal models of colitis. ${ }^{25,30} \mathrm{Good}$ evidence exists that targeting IL-1 $\beta$ pathways could be beneficial in IBD. ${ }^{31,32}$ DCAMs directly induce IL-10 secretion in macrophages and enhance IL-10 expression during innate immune activation through TLR4. Mouse studies have demonstrated that IL-10 is a critical component of mucosa protection from exacerbated inflammation after epithelial barrier disruption or aberrant T-cell activation. ${ }^{33}$ Although clinical improvement has been seen in patients with mild to moderate CD receiving recombinant human IL-10, its effect may be limited due to bioavailability and compartmentalization ${ }^{34}$ and the concomitant induction of proinflammatory IFN- $\gamma$ expression in patients with active chronic CD. ${ }^{35}$ Interestingly, DCAMs did not induce IFN-c or IL-2 secretion by T cells during antigen-specific antigen presentation in vitro (data not shown).

Our findings are of particular significance for potential therapeutic intervention in $\mathrm{CD}$, in which a loss of synergy between NOD2 and TLR signaling has been associated with an imbalance between inhibitory and proinflammatory immune effector secretion. The 3020insC mutation of NOD2 found in CD patients results in a protein product that no longer reacts to MDP to drive NF- $\kappa$ B signaling. ${ }^{36-38}$ However; it is unclear how this loss of function mutation can associate with an increased inflammatory response in patients. One explanation may be that tissues isolated from CD patients show depressed IL-10 production and that cells homozygous for the 3020insC mutation release less IL-10 after stimulation with TLR2 ligands. ${ }^{39,40}$ TLR and NOD2 signals together can induce IL-10 production when macrophages are exposed to PNCV, a crude membrane preparation of $S$. pneumoniae. ${ }^{41}$ Recently, it has been demonstrated that the 3020insC variation of NOD2 fails to activate p38, MAPK, and a key transcriptional regulator that drives IL-10 expression. ${ }^{42}$ Enhancing IL-10 production, which can occur after treatment with probiotic bacteria, may help to reduce inflammation in $\mathrm{CD} .{ }^{39}$

Collectively, we have generated new small molecule inhibitors that protect mice from severe colitis and promote mucosal recovery by enhancing protective cytokine production, while inhibiting proinflammatory stimuli during microbial recognition. The unique ability to enhance IL-10 expression and reduced TNF- $a$ production together make DCAMs promising candidates for normalizing the exacerbated innate immune responses that are maintained by microbial signals in IBD.

\section{Acknowledgments}

We thank Dr. Atul K. Bhan, Professor of Pathology, Harvard Medical School and Massachusetts General Hospital for providing expertise in scoring the histology slides and Susan Davis for help with the preparation of the article. 


\section{References}

1. Podolsky DK. Inflammatory bowel disease (1). N Engl J Med. 1991; 325:928-937. [PubMed: 1881418]

2. Strober W, Fuss I, Mannon P. The fundamental basis of inflammatory bowel disease. J Clin Invest. 2007; 117:514-521. [PubMed: 17332878]

3. Strober W, Fuss IJ, Blumberg RS. The immunology of mucosal models of inflammation. Annu Rev Immunol. 2002; 20:495-549. [PubMed: 11861611]

4. Shanahan F. Crohn's disease. Lancet. 2002; 359:62-69. [PubMed: 11809204]

5. Franchi L, Park JH, Shaw MH, et al. Intracellular NOD-like receptors in innate immunity, infection and disease. Cell Microbiol. 2008; 10:1-8. [PubMed: 17944960]

6. Sirard JC, Vignal C, Dessein R, et al. Nod-like receptors: cytosolic watchdogs for immunity against pathogens. PLoS Pathog. 2007; 3:e152. [PubMed: 18166077]

7. Akira S. Toll-like receptor signaling. J Biol Chem. 2003; 278:38105-38108. [PubMed: 12893815]

8. Akira S, Uematsu S, Takeuchi O. Pathogen recognition and innate immunity. Cell. 2006; 124:783801. [PubMed: 16497588]

9. Takeda K, Akira S. Toll-like receptors in innate immunity. Int Immunol. 2005; 17:1-14. [PubMed: 15585605]

10. Rosnet O, Marchetto S, deLapeyriere O, et al. Murine Flt3, a gene encoding a novel tyrosine kinase receptor of the PDGFR/CSF1R family. Oncogene. 1991; 6:1641-1650. [PubMed: 1656368]

11. McKenna HJ. Role of hematopoietic growth factors/flt3 ligand in expansion and regulation of dendritic cells. Curr Opin Hematol. 2001; 8:149-154. [PubMed: 11303147]

12. Shaw SG, Maung AA, Steptoe RJ, et al. Expansion of functional NK cells in multiple tissue compartments of mice treated with Flt3-ligand: implications for anti-cancer and anti-viral therapy. J Immunol. 1998; 161:2817-2824. [PubMed: 9743341]

13. Antonysamy MA, Thomson AW. Flt3 ligand (FL) and its influence on immune reactivity. Cytokine. 2000; 12:87-100. [PubMed: 10671293]

14. Swee LK, Bosco N, Malissen B, et al. Expansion of peripheral naturally occurring T regulatory cells by Fms-like tyrosine kinase 3 ligand treatment. Blood. 2009; 113:6277-6287. [PubMed: 19211508]

15. Liu K, Victora GD, Schwickert TA, et al. In vivo analysis of dendritic cell development and homeostasis. Science. 2009; 324:392-397. [PubMed: 19286519]

16. Das L, DeVecchio J, Heinzel FP. Fms-like tyrosine kinase 3-based immunoprophylaxis against infection is improved by adjuvant treatment with anti-interleukin-10 antibody. J Infect Dis. 2005; 192:693-702. [PubMed: 16028140]

17. Isambert N, Campone M, Bourbouloux E, et al. Evaluation of the safety of C-1311 (SYMADEX) administered in a phase 1 dose escalation trial as a weekly infusion for 3 consecutive weeks in patients with advanced solid tumours. Eur J Cancer. 2010; 46:729-734. [PubMed: 20031390]

18. Karlik, S.; Christensen, J.; Boss, M., et al. 59th American Academy of Neurology (AAN) Annual Meeting; 2007;

19. Krieglstein CF, Anthoni C, Cerwinka WH, et al. Role of blood- and tissue-associated inducible nitric-oxide synthase in colonic inflammation. Am J Pathol. 2007; 170:490-496. [PubMed: 17255317]

20. Weischenfeldt, J. Cold Spring Harb Protoc. 2008. Bone marrow derived macrophages (BMM): isolation and applications.

21. Burli RW, Haughan AF, Hodges AJ. Development of small-molecule therapies for autoimmune diseases. Autoimmunity. 2010; 43:526-538. [PubMed: 20388059]

22. Boonstra A, Rajsbaum R, Holman M, et al. Macrophages and myeloid dendritic cells, but not plasmacytoid dendritic cells, produce IL-10 in response to MyD88- and TRIF-dependent TLR signals, and TLR-independent signals. J Immunol. 2006; 177:7551-7558. [PubMed: 17114424]

23. Williamson E, Bilsborough JM, Viney JL. Regulation of mucosal dendritic cell function by receptor activator of NF-kappa B (RANK)/ RANK ligand interactions: impact on tolerance induction. J Immunol. 2002; 169:3606-3612. [PubMed: 12244151] 
24. Weber B, Saurer L, Mueller C. Intestinal macrophages: differentiation and involvement in intestinal immunopathologies. Semin Immunopathol. 2009; 31:171-184. [PubMed: 19533135]

25. Reinecker HC, Steffen M, Witthoeft T, et al. Enhanced secretion of tumour necrosis factor alpha, IL-6, and IL-1 beta by isolated lamina propria mononuclear cells from patients with ulcerative colitis and Crohn's disease. Clin Exp Immunol. 1993; 94:174-181. [PubMed: 8403503]

26. Sanchez-Munoz F, Dominguez-Lopez A, Yamamoto-Furusho JK. Role of cytokines in inflammatory bowel disease. World J Gastroenterol. 2008; 14:4280-4288. [PubMed: 18666314]

27. Zabana Y, Domenech E, Manosa M, et al. Infliximab safety profile and long-term applicability in inflammatory bowel disease: 9-year experience in clinical practice. Aliment Pharmacol Ther. 2010; 31:553-560. [PubMed: 20002026]

28. Danese S, Semeraro S, Armuzzi A, et al. Biological therapies for inflammatory bowel disease: research drives clinics. Mini Rev Med Chem. 2006; 6:771-784. [PubMed: 16842127]

29. Ito H, Takazoe M, Fukuda Y, et al. A pilot randomized trial of a human anti-interleukin-6 receptor monoclonal antibody in active Crohn's disease. Gastroenterology. 2004; 126:989-996. discussion 947. [PubMed: 15057738]

30. Ligumsky M, Simon PL, Karmeli F, et al. Role of interleukin 1 in inflammatory bowel diseaseenhanced production during active disease. Gut. 1990; 31:686-689. [PubMed: 2379873]

31. Cominelli F, Pizarro TT. Interleukin-1 and interleukin-1 receptor antagonist in inflammatory bowel disease. Aliment Pharmacol Ther. 1996; 10(suppl 2):49-53. discussion 54. [PubMed: 8899101]

32. Shigematsu S. Therapeutic potential of interleukin-1 receptor antagonist in inflammatory bowel disease. Kurume Med J. 1998; 45:175-179. [PubMed: 9715044]

33. Madsen KL, Malfair D, Gray D, et al. Interleukin-10 gene-deficient mice develop a primary intestinal permeability defect in response to enteric microflora. Inflamm Bowel Dis. 1999; 5:262270. [PubMed: 10579119]

34. Fedorak RN, Gangl A, Elson CO, et al. The Interleukin 10 Inflammatory Bowel Disease Cooperative Study Group. Recombinant human interleukin 10 in the treatment of patients with mild to moderately active Crohn's disease. Gastroenterology. 2000; 119:1473-1482. [PubMed: 11113068]

35. Tilg H, van Montfrans C, van den Ende A, et al. Treatment of Crohn's disease with recombinant human interleukin 10 induces the proinflammatory cytokine interferon gamma. Gut. 2002; 50:191-195. [PubMed: 11788558]

36. Girardin SE, Boneca IG, Viala J, et al. Nod2 is a general sensor of peptidoglycan through muramyl dipeptide (MDP) detection. J Biol Chem. 2003; 278:8869-8872. [PubMed: 12527755]

37. Inohara N, Nunez G. NODs: intracellular proteins involved in inflammation and apoptosis. Nat Rev Immunol. 2003; 3:371-382. [PubMed: 12766759]

38. Faustin B, Lartigue L, Bruey JM, et al. Reconstituted NALP1 inflammasome reveals two-step mechanism of caspase-1 activation. Mol Cell. 2007; 25:713-724. [PubMed: 17349957]

39. Baumgart DC, Sandborn WJ. Inflammatory bowel disease: clinical aspects and established and evolving therapies. Lancet. 2007; 369:1641-1657. [PubMed: 17499606]

40. Netea MG, Kullberg BJ, de Jong DJ, et al. NOD2 mediates anti-inflammatory signals induced by TLR2 ligands: implications for Crohn's disease. Eur J Immunol. 2004; 34:2052-2059. [PubMed: 15214053]

41. Moreira LO, El Kasmi KC, Smith AM, et al. The TLR2-MyD88- NOD2-RIPK2 signalling axis regulates a balanced pro-inflammatory and IL-10-mediated anti-inflammatory cytokine response to Gram-positive cell walls. Cell Microbiol. 2008; 10:2067-2077. [PubMed: 18549453]

42. Noguchi E, Homma Y, Kang X, et al. A Crohn's disease-associated NOD2 mutation suppresses transcription of human IL10 by inhibiting activity of the nuclear ribonucleoprotein hnRNP-A1. Nat Immunol. 2009; 10:471-479. [PubMed: 19349988] 


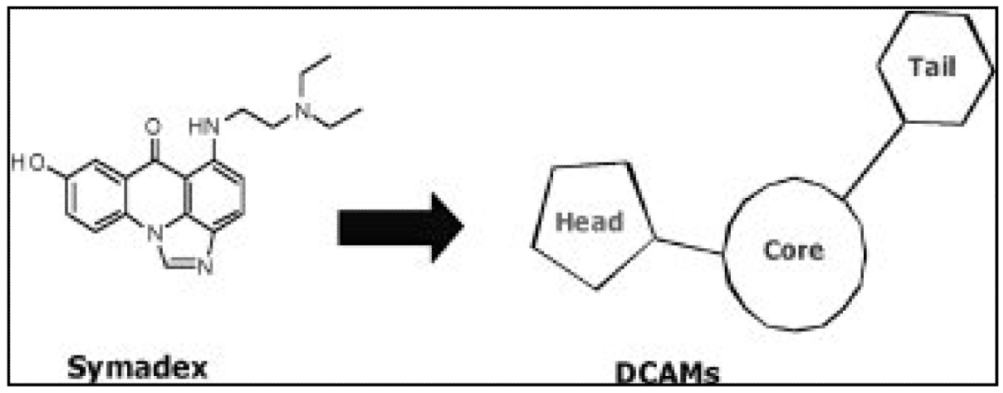

FIGURE 1.

Chemical development of DCAMs as derivatives based on the imidazoacridinone scaffold of Symadex. 


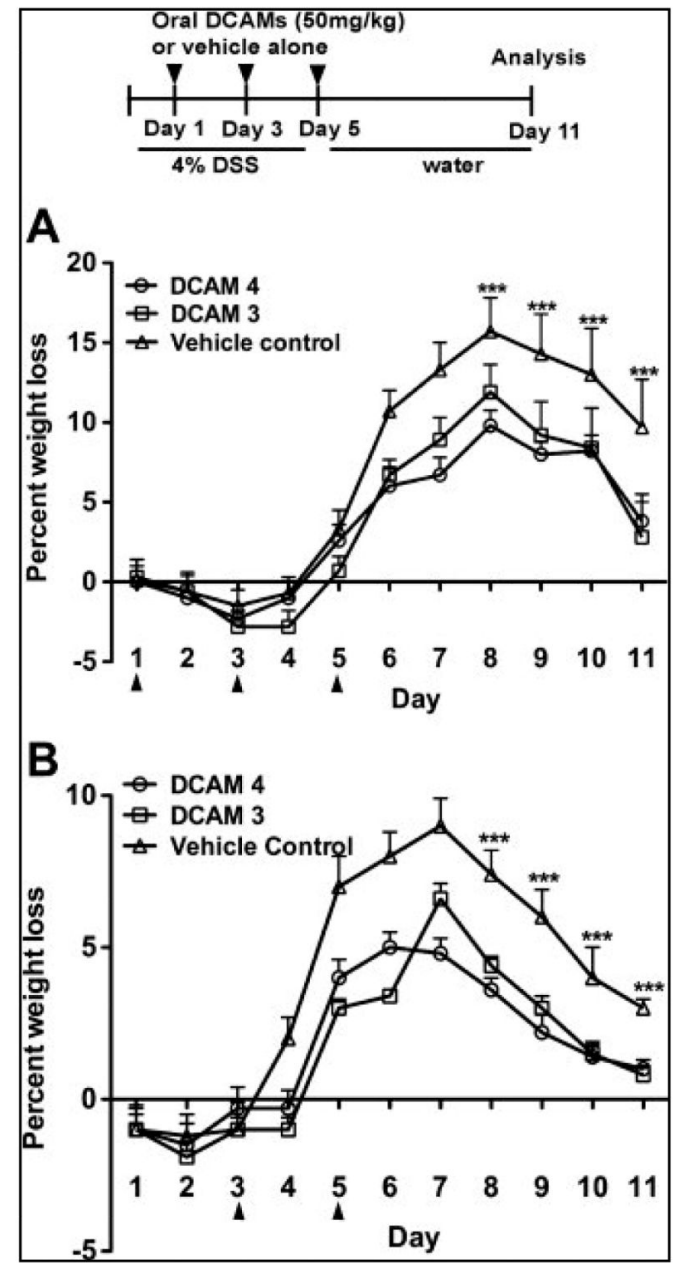

FIGURE 2.

Oral treatment with DCAMs protects from microbial-induced intestinal inflammation during DSS-induced colitis. Female C57BL/6 mice ( $n=5 /$ group) were administered three oral doses (A) or two oral doses (B) of respective compounds or vehicle control. Mice were monitored for weight loss or absence thereof. Percent weight loss was calculated and plotted. Representative experiments are shown (error bars represent standard deviation and $* * * \mathrm{P}<0.001)$. 


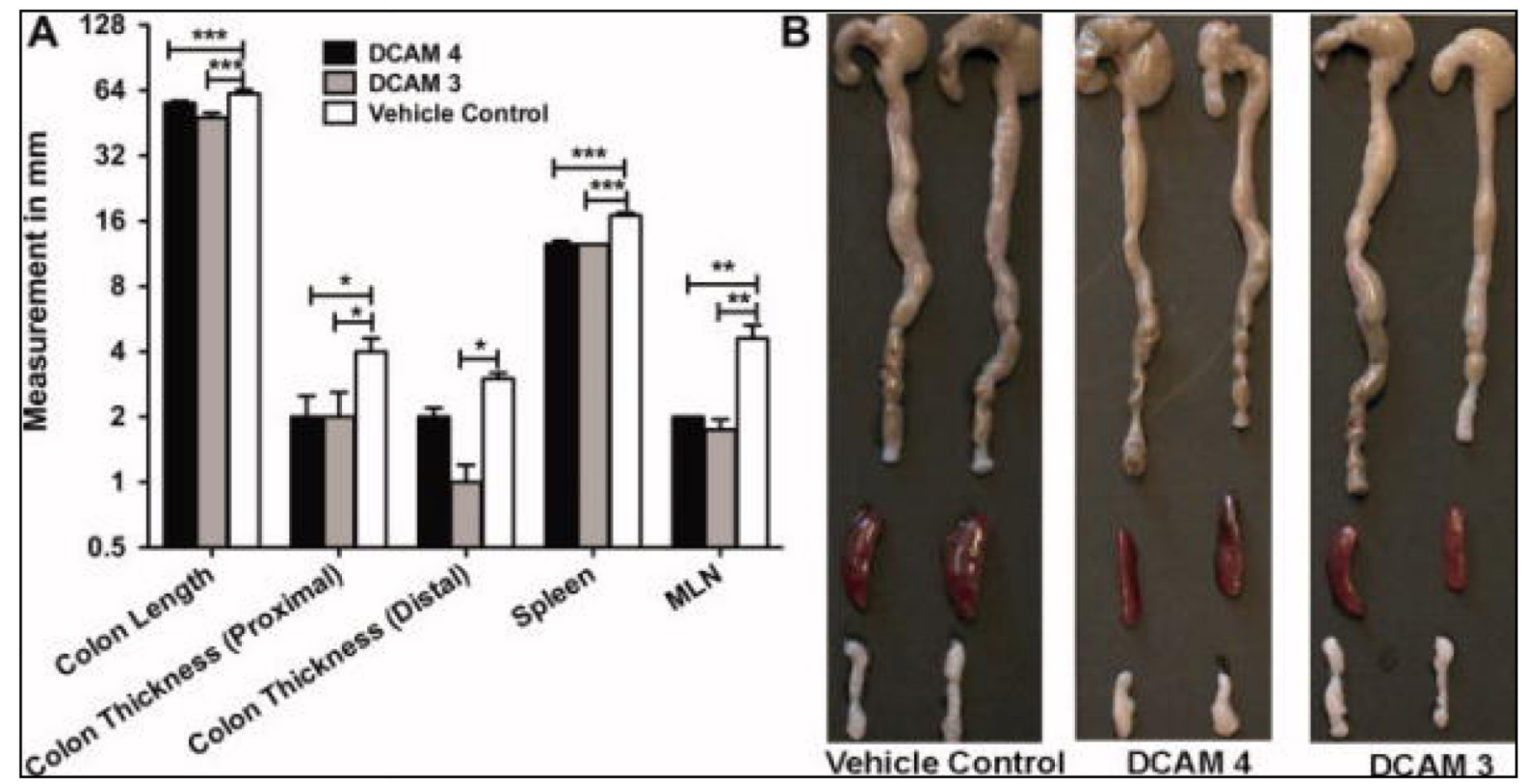

FIGURE 3.

DCAMs reduce inflammatory responses in spleen and mucosal immune system. (A) Tissues were harvested from mice treated with three doses of compounds and analyzed for colon length and thickening, enlarged spleen, and MLN. (B) Macroscopic and gross examination of colon, spleen, and MLNs harvested from mice treated with three doses of DCAM4, DCAM3, or vehicle control (*P P P $<0.001$ ). [Color figure can be viewed in the online issue, which is available at wileyonlinelibrary.com.] 


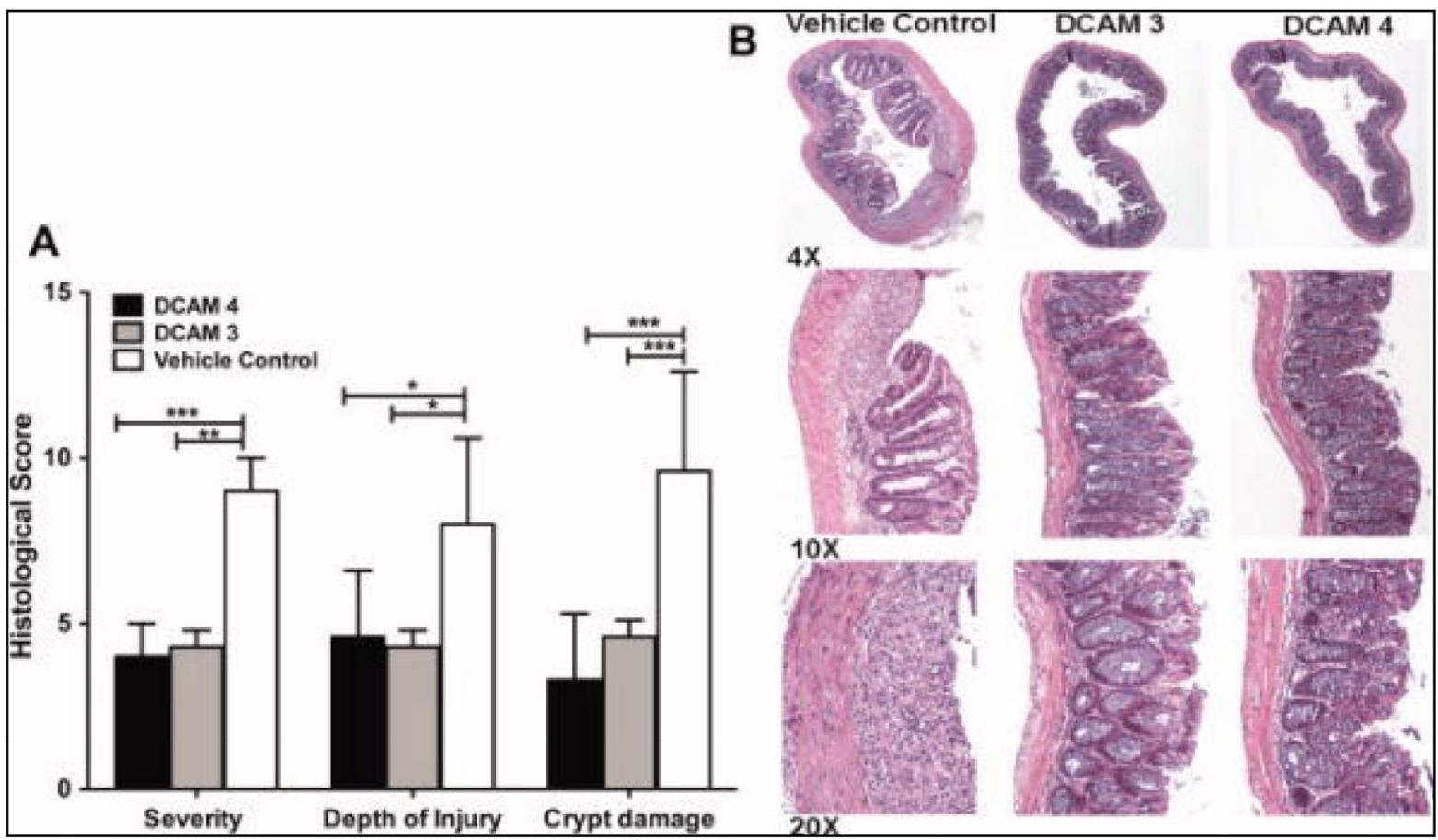

FIGURE 4.

DCAMs support mucosal recovery from severe DSS-induced colitis. (A) Colon sections were scored for three independent parameters in a blinded fashion as described in Materials and Methods. Histological scores were plotted and the error bars indicate standard deviation. (B) Histological analysis of colons harvested from mice treated with three doses of DCAM4, DCAM3, and vehicle alone. Cross-sections were stained with H\&E and examined for transepithelial neutrophil infiltration, goblet cell hyperplasia, and tissue destruction. Representative colon sections are shown here in $4 \times, 10 \times$, and $20 \times$ magnifications (*P P P < 0.001). [Color figure can be viewed in the online issue, which is available at wileyonlinelibrary.com.] 


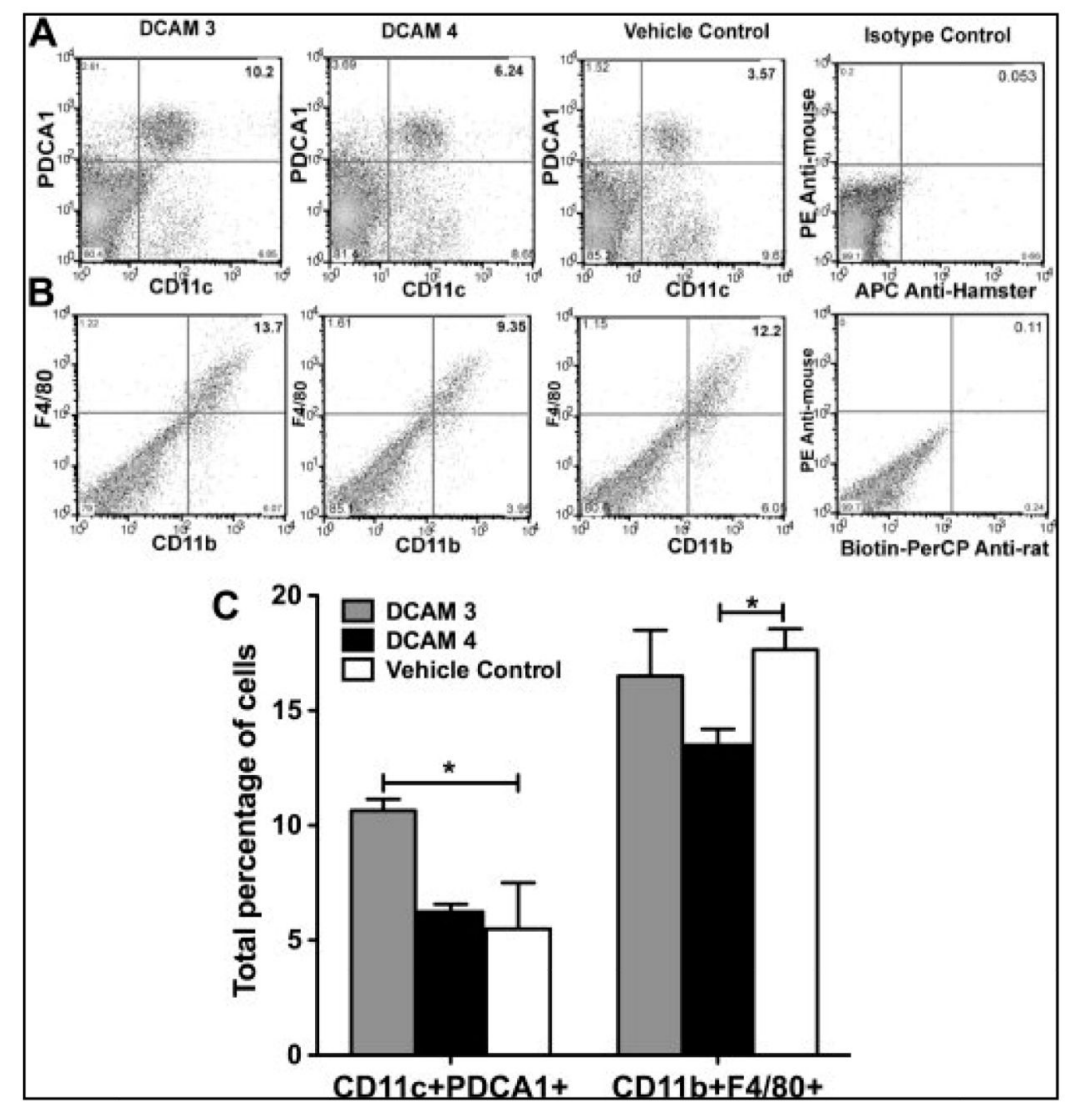

FIGURE 5.

DCAMs regulate DC and macrophage subsets during mucosal recovery. Characterization of the DC population from colons harvested after treatment with DCAM3, DCAM4, or vehicle control. FACS analysis of populations CD11c and PDCA1 (A), CD11b and F4/80 (B), and bar graph representation of the percentage of cells analyzed using FlowJo software (C). Values represent means from two independent experiments $(* \mathrm{P}<0.05)$. 


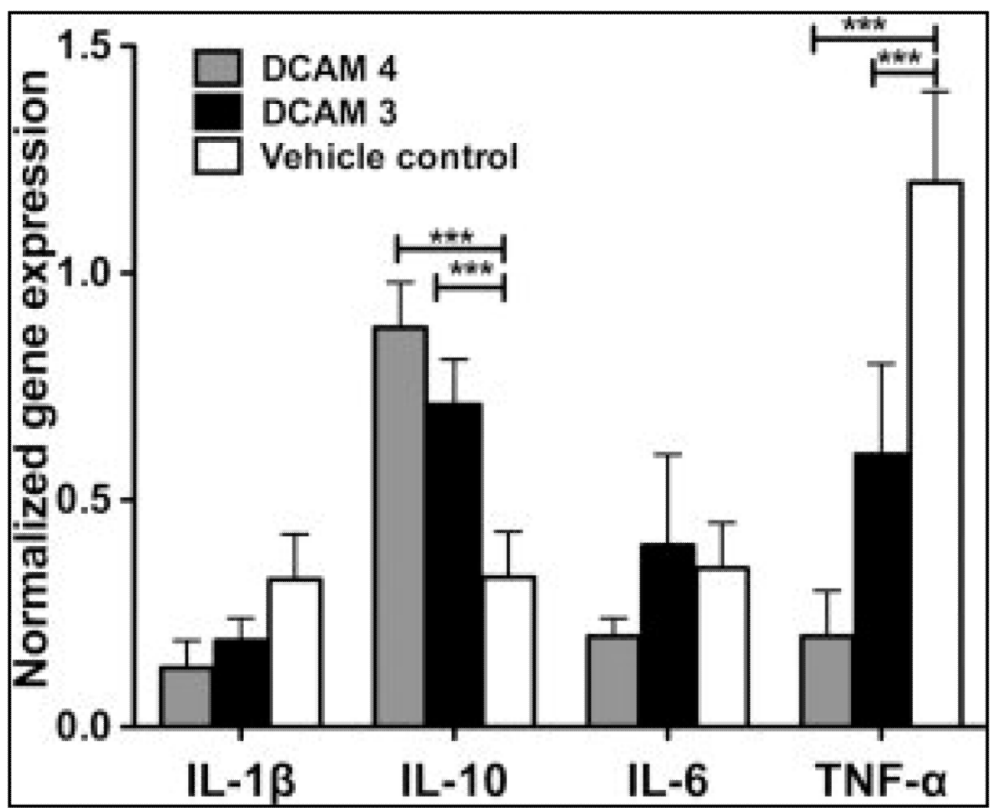

FIGURE 6.

DCAMs enhance inhibitory and reduce proinflammatory cytokines expression in the mucosal immune system. Quantitative PCR analysis of MLNs for cytokine gene expression of IL-10, IL-6, IL-1[beta], and TNF-[alpha]. MLNs were harvested from mice treated with respective compounds or vehicle control and were used to study cytokine gene expression by quantitative PCR. Error bars represent the standard error of mean values. Representative data are shown $(* * * \mathrm{P}<0.001)$. 

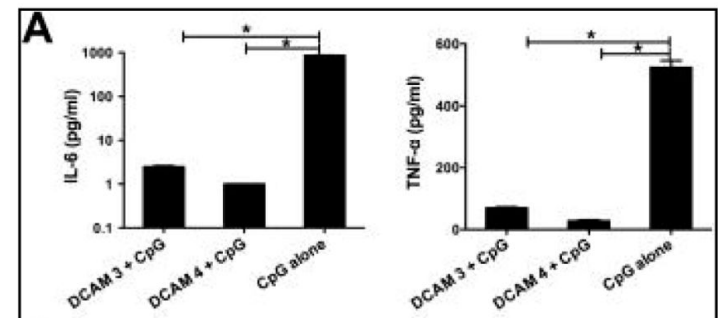

B
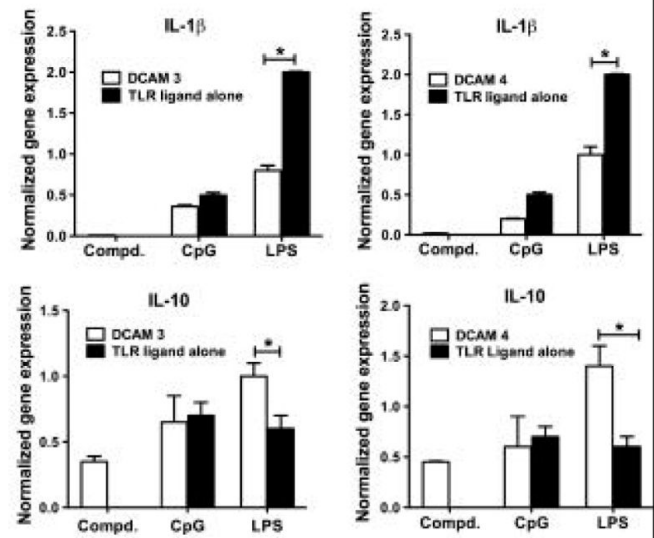

C

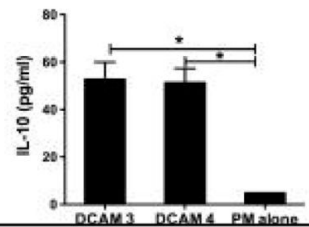

FIGURE 7.

DCAMs promote inhibitory innate immune responses. (A) In vitro cytokine production of IL-6 and TNF-[alpha] by CpG activated BMM in the presence of DCAM-3 or DCAM-4 as measured by Luminex. (B) Quantitative PCR analysis of IL-10 and IL-1[beta] gene expression by TLR-activated BMMs exposed to either DCAM4 or DCAM3. Relative gene expression was calculated with GAPDH as the reference gene. (C) In vitro IL-10 production by peritoneal macrophages in the presence of DCAM-3 or DCAM-4. Representative experiments are shown with error bars indicating standard error of mean values $(* \mathrm{p}<0.05)$. 
TABLE 1

Properties of DCAMs 3 and 4

\begin{tabular}{|c|c|c|}
\hline & DCAM 3 & DCAM 4 \\
\hline \multicolumn{3}{|l|}{ Kinase assay } \\
\hline Flt3 (wt) kinase IC50 (nM) & 11 & 27 \\
\hline Flt3 (D835Y) kinase IC50 (nM) & 2 & 4 \\
\hline \multicolumn{3}{|l|}{ Off-target hits } \\
\hline $\mathrm{IC}_{50}$ range $1-50 \mathrm{nM}( \pm 25 \mathrm{nM})$ & ROS1 & ROS1 \\
\hline $\mathrm{IC}_{50}$ range $100-500 \mathrm{nM}( \pm 25 \mathrm{nM})$ & $\begin{array}{l}\text { ALK2, CDK2, CDK5, CDK8, CLK4, CSF1R, } \\
\text { FGR, PDGFR, MUSK, NTRK1, NTRK3 }\end{array}$ & ALK2, CLK4, NTRK2, NTRK3 \\
\hline \multicolumn{3}{|l|}{ Safety } \\
\hline hERG $\operatorname{IC}_{50}(\mu \mathrm{M})$ & $>10$ & $>10$ \\
\hline 5-Strain AMES (mutagenicity) & Negative & Negative \\
\hline GPCR panel $(<50 \%$ inhib. at $10 \mu \mathrm{M})$ & 5-HT2A, Al, A3 & 5-HT2A \\
\hline Ion channel ( $<50 \%$ inhib. at $10 \mu \mathrm{M})$ & None & None \\
\hline \multicolumn{3}{|l|}{ Physical properties } \\
\hline PAMPA GIT, pH $5(\log P e)$ & -3.2 & -3.6 \\
\hline PAMPA GIT, pH $6.2(\log \mathrm{Pe})$ & -3.1 & -3.6 \\
\hline PAMPA GIT, pH $7.4(\log P e)$ & -3.2 & -4.0 \\
\hline PAMPA BBB, pH $7.4(\log P e)$ & -4.3 & -4.4 \\
\hline Mol Wt & 359 & 371 \\
\hline $\operatorname{clog} \mathrm{P}$ & 3.5 & 3.3 \\
\hline $\operatorname{clog} S$ & -5.9 & -5.7 \\
\hline cSlogP & 3.7 & 3.6 \\
\hline TPSA $\left(\AA^{2}\right)$ & 93.5 & 84.3 \\
\hline Chiral center count & 0 & 0 \\
\hline H-Bond acceptor count & 8 & 7 \\
\hline H-Bond donor count & 2 & 2 \\
\hline Rule-of-five compliant & Yes & Yes \\
\hline \multicolumn{3}{|l|}{ Metabolic stability in human hepatocytes } \\
\hline $\mathrm{T} 1 / 2(\min )$ & 69 & $>180$ \\
\hline \multicolumn{3}{|l|}{ PK (mouse, IV=5mg/kg, PO=50mg/kg) } \\
\hline $\mathrm{PO}, \mathrm{Tl} / 2(\mathrm{hr})$ & 5 & 6 \\
\hline Oral bioavailability $(\% \mathrm{~F})$ & 55 & 35 \\
\hline Vdss (mL) & 540 & 4410 \\
\hline PO clearance (mL/min) & 5 & 43 \\
\hline
\end{tabular}

